\title{
DYNAMIC PHASE-FIELD FRACTURE WITH A FIRST-ORDER DISCONTINUOUS GALERKIN METHOD FOR ELASTIC WAVES
}

\author{
Kerstin Weinberg ${ }^{1}$ And Christian Wieners ${ }^{2}$
}

\begin{abstract}
We present a new numerical approach for wave induced dynamic fracture. The method is based on a discontinuous Galerkin approximation of the first-order hyperbolic system for elastic waves and a phase-field approximation of brittle fracture driven by the maximum tension. The algorithm is staggered in time and combines an implicit midpoint rule for the wave propagation followed by an implicit Euler step for the phase-field evolution. At fracture, the material is degraded, and the waves are reflected at the diffusive interfaces. Two and three-dimensional examples demonstrate the advantages of the proposed method for the computation of crack growth and spalling initiated by reflected and superposed waves.
\end{abstract}

September 21, 2021.

\section{INTRODUCTION}

Dynamic fracture and fragmentation of solids attract constant attention from scientists, both out of simple curiosity, technological interest, and numerical challenges. Consequently, numerous computational fracture schemes have been designed, including classical discontinuous methods, such as interface formulations or local enrichment strategies, and continuous methods with discontinuities replaced by sharp gradients. The phasefield approach to fracture, chosen here, falls into the latter category and implicitly represents the cracks by an additional continuous field, an order parameter indicating the transition from elasticity to fracture. As a result, the fracture evolves naturally within the numerical computation.

Numerical schemes for dynamic phase-field fracture are well established, see, e.g., [Miehe et al., 2010, Borden et al., 2012, Hesch and Weinberg, 2014], and, more recently, [Ren et al., 2019, Mandal et al., 2020], and the references therein for an overview. They all have in common that they use the classical description of motion derived from the conservation of linear momentum, which results in a second-order partial differential equation. Their integration in time is performed with finite differences or a Newmark family method, which inevitably introduces some numerical dissipation [Hughes, 1987]. In sudden rupture or for impact problems, this spurious energy loss is usually manageable or insignificant. However, for the propagation of waves, their reflection, transmission, and superposition, the transported energy is significant, and a spurious dissipation no longer allows for a meaningful evaluation of the results. Special integration techniques try to circumvent these spurious effects, but ultimately energy loss is always a problem.

In order to solve this problem, we propose here an approach that has already proven successful in calculating conservation laws. For this purpose, the elastic equation of motion is transformed into a coupled system of firstorder differential equations, suitably discretized, and combined with a phase-field method to calculate the crack initiation and propagation. The use of first-order discontinuous Galerkin methods combined with implicit timestepping methods for the elastic wave propagation is motivated by the high accuracy, the energy conservation as long no cracks open, and the small numerical dissipation of this method. We show in our examples that these properties are essential to simulate fracture initiated by the superposition and reflection of waves. Nevertheless, these effects can be computed only with a high numerical expense on fine meshes, so that efficient parallel finite element software, including fast iterative solution methods in every implicit time step, is an indispensable requirement.

This paper is organized as follows. In Section 2, we define a phase-field model for dynamic fracture, where the phase-field evolution is driven by a stress-based fracture criterion [Bilgen and Weinberg, 2019] and where

Keywords and phrases: discontinuous Galerkin finite elements, linear wave equation, phase field, dynamic fracture, spalling

${ }^{1}$ Chair of Solid Mechanics, University of Siegen, Siegen, Germany. Email: kerstin.weinberg@uni-siegen.de

${ }^{2}$ Institute of Applied and Numerical Mathematics, KIT, Karlsruhe, Germany. Email: christian.wieners@kit.edu 
the crack is approximated by a material degradation depending on the phase field. For the discrete phasefield evolution, we use lowest order conforming finite elements in space and the implicit Euler method in time. Then, in Section 3, we recall the discontinuous Galerkin (DG) approximation for linear waves [Hesthaven and Warburton, 2008], based on the formulation of a first-order hyperbolic system and using full upwind flux [Hochbruck et al., 2015]. This DG approximation is combined with the energy-conserving implicit midpoint rule in time. Then, in Section 2, we introduce a fully coupled algorithm combining the DG method with the phase field and fracture evolution in a staggered scheme with variable time steps depending on the fracture evolution.

Our numerical experiments in Section 5 are motivated by an Hopkinson bar experiment. In the first example, we study a configuration in two dimensions, where the crack is initiated by the reflection and superposition of two incoming pressure waves. We show that our staggered scheme reproduces identical dynamic crack behavior on different meshes, indicating convergence of the numerical method. Then, this test is transferred to a threedimensional configuration which also shows crack opening by the superposition of two incoming wave signals. All numerical results are realized within the parallel finite element system $\mathrm{M}++$ [Wieners, 2010, Baumgarten and Wieners, 2021]. In Section 4, we conclude with a discussion on variational formulations of the phase-field driving forces and possible extensions of our computational approach to dynamic models for non-linear elastic and dispersive waves.

\section{A PHASE-FIELD APPROACH FOR DYNAMIC FRACTURE}

Physically, the initiation and propagation of cracks depend on various conditions, such as the geometry of the structure, the material toughness, the loading rate, the loading magnitude and its distribution. In classical fracture mechanics, the stress state at the crack tip determines the steady or unsteady crack growth [Anderson, 2004]. Following the brittle fracture approaches of Griffith and Irwin [Griffith, 1921, Irwin, 1958], the material fails when the energy release reaches a critical value $G_{\mathrm{c}}$. This assumption provides a criterion for crack growth; however, it needs to be embedded into an energy minimization setting for the entire structure to determine crack propagation, paths, and branching.

The non-local approach using a phase field goes back to the fundamental variational model of Francfort and Marigo [Francfort and Marigo, 1998,Bourdin et al., 2008] and has gained much attention recently, cf. [Henry and Levine, 2004, Miehe et al., 2010, Borden et al., 2012, Ambati and Lorenzis, 2016, Negri, 2016]. The phase-field crack modeling is popular mainly because of its potential for capturing the evolution of complex crack patterns without the need for specialized crack-front tracking algorithms.

In this section, we introduce the notation, and we summarize the model and its phase-field approximation, which builds the basis for our numerical realization.

\subsection{A dynamic model for crack evolution at small strains}

We consider a bounded Lipschitz domain $\Omega \subset \mathbb{R}^{d}$ with boundary $\partial \Omega=\bar{\Omega} \backslash \Omega$ and the time interval $[0, T]$. We want to determine the evolution of a crack in time $t \longmapsto \Gamma_{\mathrm{c}}(t) \subset \Omega$ together with the dynamics of elastic waves described by the velocity vector $\mathbf{v}$ and the stress tensor $\boldsymbol{\sigma}$

$$
\varrho \dot{\mathbf{v}}(t)=\operatorname{div} \boldsymbol{\sigma}(t)+\mathbf{f}(t), \quad \dot{\boldsymbol{\sigma}}(t)=\mathbf{C} \boldsymbol{\varepsilon}(\mathbf{v}(t)) \quad \text { in } \Omega \backslash \Gamma_{\mathrm{c}}(t)
$$

Here, $\varrho$ is the mass density, $\mathbf{f}(t)$ the applied volume force and $\mathbf{C}$ the Hookean elasticity tensor with Lamé constants $\lambda$ and $\mu$. Furthermore, for the combined model we assume that

- the crack evolution is irreversible, i.e., $\Gamma_{\mathrm{c}}\left(t_{1}\right) \subset \Gamma_{\mathrm{c}}\left(t_{2}\right) \subset \Omega$ for $t_{1}<t_{2}$;

- the elastic solution remains admissible with respect to a suitable fracture criterion;

- the crack evolution is determined by an energy dissipation criterion.

The wave propagation described by (1) is driven by initial and boundary conditions on $\partial \Omega=\partial_{\mathrm{N}} \Omega \cup \partial_{\mathrm{D}} \Omega$ together with free Neumann boundary conditions at the crack interface, i.e.,

$$
\begin{aligned}
\mathbf{v}(0) & =\mathbf{v}_{0} & \text { and } & \boldsymbol{\sigma}(0)=\boldsymbol{\sigma}_{0} \text { in } \Omega \\
\boldsymbol{\sigma}(t) \mathbf{n} & =\mathbf{g}_{\mathrm{N}}(t) \text { on } \partial_{\mathrm{N}} \Omega & \text { and } & \mathbf{v}(t)=\mathbf{v}_{\mathrm{D}}(t) \text { on } \partial_{\mathrm{D}} \Omega \\
\boldsymbol{\sigma}(t) \mathbf{n} & =\mathbf{0} \text { on } \Gamma_{\mathrm{c}}(t) . & &
\end{aligned}
$$

The configuration depends on initial data $\mathbf{v}_{0}$ and $\boldsymbol{\sigma}_{0}$, volume forces $\mathbf{f}$ in $\Omega$, and boundary data $\mathbf{g}_{\mathrm{N}}$ and $\mathbf{v}_{\mathrm{D}}$. The model has to be complemented by an evolution law for $\Gamma_{c}$. 
Energy dissipation. At time $t$, the mechanical energy of the first-order system is given by

$$
\mathcal{E}(t)=\frac{1}{2} \int_{\Omega \backslash \Gamma_{\mathrm{c}}(t)}\left(\varrho|\mathbf{v}(t)|^{2}+\boldsymbol{\sigma}(t) \cdot \mathbf{C}^{-1} \boldsymbol{\sigma}(t)\right) \mathrm{d} \mathbf{x} .
$$

In the case of homogeneous data, $\mathbf{f}=\mathbf{0}$ and $\mathbf{g}_{\mathrm{N}}=\mathbf{0}$, we have $\mathcal{E}\left(t_{1}\right)=\mathcal{E}\left(t_{2}\right)$ if $\Gamma_{\mathrm{c}}\left(t_{1}\right)=\Gamma_{\mathrm{c}}\left(t_{2}\right)$. In the case of crack propagation, $\Gamma_{\mathrm{c}}\left(t_{2}\right) \backslash \Gamma_{\mathrm{c}}\left(t_{1}\right) \neq \emptyset$, we claim for energy dissipation,

$$
\begin{aligned}
\mathcal{D}\left(t_{1}, t_{2}\right) & =\mathcal{E}\left(t_{1}\right)-\mathcal{E}\left(t_{2}\right) \\
& =G_{\mathrm{c}}\left|\Gamma_{\mathrm{c}}\left(t_{2}\right) \backslash \Gamma_{\mathrm{c}}\left(t_{1}\right)\right|_{d-1}, \quad t_{1}<t_{2},
\end{aligned}
$$

i.e., the energy release is proportional to the (scaled) $d-1$ surface volume of the crack increment, where the Griffiths constant $G_{\mathrm{c}}>0$ is a material parameter determining the critical energy release rate. Energy dissipation in a dynamic phase-field approximation can be included by a minimization formulation in every time step [Larsen et al., 2010]. Here we propose a different approach where energy dissipation is integrated in the time-stepping scheme; details will be outlined in Section 4.

Crack criteria. To formulate a fracture evolution criterion we use here the maximum principal stress

$$
\sigma_{\mathrm{I}}=\max _{\mathbf{n} \in S^{2}} \mathbf{n} \cdot \boldsymbol{\sigma n}, \quad S^{2}=\left\{\mathbf{n} \in \mathbb{R}^{3}:|\mathbf{n}|=1\right\}
$$

and relate it to a material resistance value $\sigma_{\mathrm{c}}>0$. We assume that the material response is elastic, if for all $\mathbf{n} \in S^{2}$ the tension $\boldsymbol{\sigma} \mathbf{n}$ in direction $\mathbf{n}$ is smaller than $\sigma_{\mathrm{c}}$. Otherwise, if the maximum principal stress gets larger, i.e., $\sigma_{\mathrm{I}}(t, \mathbf{x}) \geq \sigma_{\mathrm{c}}$, a driving force is activated which initiates fracture.

Note that the stress-based criterion only activates fracture driving forces in our dynamic model, and sufficiently strong forces are required to open a crack. This criterion is different from a quasi-static model with pointwise constraint $\sigma_{\mathrm{I}} \leq \sigma_{\mathrm{c}}$ which results in an instantaneous nonlinear material response and thus in a faster material response than wave propagation.

\subsection{A phase-field approximation}

The crack evolution is described by an order parameter, the phase field

$$
s:[0, T] \times \bar{\Omega} \longrightarrow[0,1],
$$

starting with $s(0, \mathbf{x})=1$ for $\mathbf{x} \in \Omega$. In our approach, the phase-field evolution is reversible, so that in the case that the elastic driving force is not strong enough to initiate fracture, the phase field recovers if the elastic driving force is getting smaller. The irreversible physical process of crack opening depends on the history, the infimum phase field

$$
s_{\text {inf }}(t, \mathbf{x})=\inf \left\{s\left(t^{\prime}, \mathbf{x}\right): 0 \leq t^{\prime} \leq t\right\},
$$

determing the elastic domain

$$
\Omega\left(s_{\text {inf }}\right)=\left\{\mathbf{x} \in \Omega: s_{\text {inf }}(t, \mathbf{x})>s_{\min }\right\} .
$$

Depending on the critical parameter $s_{\min }>0$ this defines the phase-field approximation of the fracture zone $\Omega \backslash \Omega\left(s_{\text {inf }}\right)$. Note that this parameter is a regularization which extends the fracture $\Gamma_{\mathrm{c}}(t)$ to a volume $\Omega \backslash \Omega\left(s_{\text {inf }}\right)$. Depending on the phase field $s$ at time $t \in[0, T]$, the crack surface energy is approximated by

$$
\mathcal{G}(s)=\frac{G_{\mathrm{c}}}{l_{\mathrm{c}}} \int_{\Omega} \gamma_{\mathrm{c}}(s) \mathrm{d} \mathbf{x} \text { with } \quad \gamma_{\mathrm{c}}(s)=\frac{1}{2}(s-1)^{2}+\frac{l_{\mathrm{c}}^{2}}{2}|\nabla s|^{2},
$$

depending on the Griffiths constant $G_{\mathrm{c}}>0$ and a small length scale parameter $l_{\mathrm{c}}>0$ which determines the width of the diffusive interface [Pandolfi et al., 2021]. 
Phase-field evolution. The initiation and propagation of phase-field fracture is modeled by the following principles:

- The crack evolution depends on a retardation time $\tau_{\mathrm{r}}>0$ and a driving force $Y$, i.e.,

$$
\tau_{\mathrm{r}} \dot{s}=-Y(\boldsymbol{\sigma}, s) \quad \text { in }(0, T) \times \Omega
$$

- The driving force is composed of the elastic driving force $Y_{\mathrm{el}}$ and a geometric term $Y_{\text {geom }}$ corresponding to the crack surface energy

$$
Y(\boldsymbol{\sigma}, s)= \begin{cases}Y_{\mathrm{el}}(\boldsymbol{\sigma})-M_{\mathrm{geom}} Y_{\text {geom }}(s) & s>0 \\ 0 & s=0\end{cases}
$$

and complemented by the admissibility conditions which enforces $s(t, \mathbf{x}) \geq 0$. This includes a scaling parameter $M_{\text {geom }}>0$ which is required for the calibration of the two components $Y_{\text {el }}$ and $Y_{\text {geom }}$ and which depends on the formulation of the crack driving force. For a comparison of different models for $Y_{\mathrm{el}}$ we refer to [Bilgen and Weinberg, 2019].

- Here, we use the stress-based criterion for the elastic driving force

$$
Y_{\mathrm{el}}(\boldsymbol{\sigma})=\max \left\{\frac{\sigma_{\mathrm{I}}}{\sigma_{\mathrm{c}}}-1,0\right\}
$$

which is active if the maximum principal stress $\sigma_{\mathrm{I}}$ exceeds the resistance value $\sigma_{\mathrm{c}}>0$.

- The geometric term $Y_{\text {geom }}$ is the gradient flow with respect to $\gamma_{c}$, i.e.,

$$
Y_{\text {geom }}(s)=1-s+l_{\mathrm{c}}^{2} \Delta s
$$

complemented with homogeneous Neumann boundary conditions $\nabla s \cdot \mathbf{n}=0$ on $\partial \Omega$. The latter is realized in weak form, i.e.,

$$
\int_{\Omega} Y_{\text {geom }}(s) \phi \mathrm{d} \mathbf{x}=\int_{\Omega}\left((1-s) \phi-l_{\mathrm{c}}^{2} \nabla s \cdot \nabla \phi\right) \mathrm{d} \mathbf{x}, \quad \phi \in \mathrm{H}^{1}(\Omega) .
$$

Altogether, we obtain for the phase-field evolution (5) the parabolic equation

$$
\tau_{\mathrm{r}} \int_{\Omega} \dot{s} \phi \mathrm{d} \mathbf{x}+M_{\text {geom }} \int_{\Omega}\left((s-1) \phi+l_{\mathrm{c}}^{2} \nabla s \cdot \nabla \phi\right) \mathrm{d} \mathbf{x}=-\int_{\Omega} Y_{\mathrm{el}}(\boldsymbol{\sigma}) \phi \mathrm{d} \mathbf{x}, \quad \phi \in \mathrm{H}^{1}(\Omega),
$$

which is complemented by a projection to ensure admissibiliy $s(\mathbf{x}) \in[0,1]$.

Material degradation. If $s(t, \mathbf{x}) \leq s_{\min }$ at a material point $\mathbf{x} \in \Omega$, then the material is cracked. Depending on the phase-field history $s_{\text {inf }}(t, \mathbf{x})$ and the elastic domain $\Omega\left(s_{\text {inf }}\right)$ the elasticity tensor at time $t$ is defined by

$$
\mathbf{C}(t, \mathbf{x})=s_{\text {inf }}\left(t^{\prime}, \mathbf{x}\right) \mathbf{C}+\left(1-s_{\text {inf }}\left(t^{\prime}, \mathbf{x}\right)\right) \mathbf{C}_{\text {reg }}, \quad t^{\prime}=\inf \left\{\tau \in[0, t]: \Omega\left(s_{\text {inf }}(\tau)\right)=\Omega\left(s_{\text {inf }}\right)\right\}
$$

with a small but positive definite tensor $\mathbf{C}_{\text {reg }}$, e.g., $\mathbf{C}_{\text {reg }}=10^{-7} \mathbf{C}$. In the phase-field approximation, $\mathbf{C}_{\text {reg }}^{-1}$ is a penalty term which enforces that the stress nearly vanishes in the crack zone. Numerically we observe that the results are not very sensitive with respect to choice of $\mathbf{C}_{\text {reg }}$.

By this construction the material only degrades when the fracture zone (determined by the parameter $s_{\text {min }}$ ) increases. In particular, the full domain is elastic, i.e., $\mathbf{C}(0)=\mathbf{C}(t)$ as long $\Omega=\Omega\left(s_{\text {inf }}\right)$.

Then, the linear wave equation $(1)$ in $\Omega \backslash \Gamma_{\mathrm{c}}(t)$ is approximated by

$$
\varrho \dot{\mathbf{v}}(t)=\operatorname{div} \boldsymbol{\sigma}(t)+\mathbf{f}(t), \quad \dot{\boldsymbol{\sigma}}(t)=\mathbf{C}(t) \boldsymbol{\varepsilon}(\mathbf{v}(t)) \quad \text { in } \Omega,
$$

and the phase-field approximation of the mechanical energy (3) is given by

$$
\mathcal{E}_{\mathrm{pf}}(t)=\frac{1}{2} \int_{\Omega}\left(\varrho|\mathbf{v}(t)|^{2}+\boldsymbol{\sigma}(t) \cdot \mathbf{C}(t)^{-1} \boldsymbol{\sigma}(t)\right) \mathrm{d} \mathbf{x}
$$

This approximates the energy dissipation

$$
\mathcal{D}_{\mathrm{pf}}\left(t_{1}, t_{2}\right)=\mathcal{E}_{\mathrm{pf}}\left(t_{1}\right)-\mathcal{E}_{\mathrm{pf}}\left(t_{2}\right) \geq 0, \quad t_{1}<t_{2},
$$

which is strictly positive in case of fracture evolution, i.e., $\mathbf{C}\left(t_{1}\right) \neq \mathbf{C}\left(t_{2}\right)$. 
Note that the degradation (9) is the most simple choice, in more general formulations a monotone increasing degradation function $g(\cdot)$ with $g(0)=0$ and $g(1)=1$ is included in (9); see, e.g., [Kuhn et al., 2015, Sargado et al., 2018] for comparing different options. Then, the degraded elasticity tensor is given by

$$
\mathbf{C}_{g}(t, \mathbf{x})=g\left(s_{\mathrm{inf}}\left(t^{\prime}, \mathbf{x}\right)\right) \mathbf{C}+\left(1-g\left(s_{\mathrm{inf}}\left(t^{\prime}, \mathbf{x}\right)\right)\right) \mathbf{C}_{\mathrm{reg}} .
$$

This can be complemented by a degradation of the density

$$
\varrho_{g}(t, \mathbf{x})=g\left(s_{\mathrm{inf}}\left(t^{\prime}, \mathbf{x}\right)\right) \varrho+\left(1-g\left(s_{\mathrm{inf}}\left(t^{\prime}, \mathbf{x}\right)\right)\right) \varrho_{\mathrm{reg}}
$$

and a moderate choice $\varrho_{\text {reg }} \in(0, \varrho]$. The limiting physical model for $\ell_{\mathrm{c}} \longrightarrow 0$ and $\mathbf{C}_{\text {reg }} \longrightarrow 0$ with fixed $\varrho_{\text {reg }}>0$ is identical for all choices of material degradation. For numerical tests with sufficiently small length scale parameters $l_{\mathrm{c}}>0$ and time steps $\Delta t>0$, see Section 5 , no qualitative improvement is observed by including different degradation functions, and so we formulate the algorithmic approach for the simple choice (9).

\section{A Runge-Kutta discontinuous Galerkin method for elastic Waves}

The linear wave equation (10) with time-dependent elasticity tensor $\mathbf{C}(t)$ is approximated with a discontinuous Galerkin (DG) method in space and a Runge-Kutta method in time, see [Hochbruck et al., 2015] for details. For hyperbolic applications, this scheme is a widely used extension of finite volume methods, see, e.g., [Dumbser and Käser, 2006]. A first-order DG scheme is less dissipative than a second-order approach and it provides also approximations in case of discontinuities of the solution. Here we shortly summarize the adoption of this method to our phase-field fracture application.

The discretization is based on a formulation of the linear first-order system (10)

$$
M(t)\left(\begin{array}{c}
\dot{\mathbf{v}}(t) \\
\dot{\boldsymbol{\sigma}}(t)
\end{array}\right)=A\left(\begin{array}{c}
\mathbf{v}(t) \\
\boldsymbol{\sigma}(t)
\end{array}\right)+\mathbf{b}(t), \quad t \in(0, T)
$$

where the energy operator $M(t)$, the differential operator $A$, and the right-hand side $\mathbf{b}$ are given by

$$
\begin{aligned}
\left(M(t)(\dot{\mathbf{v}}(t), \dot{\boldsymbol{\sigma}}(t),(\mathbf{w}, \boldsymbol{\eta}))_{0, \Omega}\right. & =(\rho \dot{\mathbf{v}}(t), \mathbf{w}(t))_{0, \Omega}+\left(\mathbf{C}(t)^{-1} \dot{\boldsymbol{\sigma}}(t), \boldsymbol{\eta}\right)_{0, \Omega}, \\
(A(\mathbf{v}(t), \boldsymbol{\sigma}(t)),(\mathbf{w}, \boldsymbol{\eta}))_{0, \Omega} & =(\operatorname{div} \boldsymbol{\sigma}(t), \mathbf{w})_{0, \Omega}+\left(\varepsilon(\mathbf{v}(t), \boldsymbol{\eta})_{0, \Omega}+(\mathbf{v}(t), \boldsymbol{\eta} \mathbf{n})_{0, \Gamma_{\mathrm{D}}}+(\boldsymbol{\sigma}(t) \mathbf{n}, \mathbf{w})_{0, \Gamma_{\mathrm{N}}}\right. \\
(\mathbf{b}(t),(\mathbf{w}, \boldsymbol{\eta}))_{0, \Omega} & =(\mathbf{f}(t), \mathbf{w})_{0, \Omega}+\left(\mathbf{v}_{\mathrm{D}}(t), \boldsymbol{\eta} \mathbf{n}\right)_{0, \Gamma_{\mathrm{D}}}+\left(\mathbf{g}_{\mathrm{N}}(t), \mathbf{w}\right)_{0, \Gamma_{\mathrm{N}}}
\end{aligned}
$$

for test functions $(\mathbf{w}, \boldsymbol{\eta})$ in $\Omega$. This defines $\mathcal{E}_{\mathrm{pf}}(t)=\frac{1}{2}\left(M(t)(\mathbf{v}(t), \boldsymbol{\sigma}(t),(\mathbf{v}(t), \boldsymbol{\sigma}(t)))_{0, \Omega}\right.$. We use the standard notation for the $\mathrm{L}_{2}$ inner products $(\cdot, \cdot)_{0, \Omega}$ and $(\cdot, \cdot)_{0, \Gamma}$ in the domain and on the boundaries.

For the approximation of (11) in space, we need to construct approximations $M_{h}, A_{h}$ and $\mathbf{b}_{h}$. On a mesh $\Omega_{h}=\bigcup_{K \in \mathcal{K}_{h}} K$ with elements $K$, let $V_{h}^{\mathrm{dg}}=\prod_{K \in \mathcal{K}_{h}} \mathbb{P}_{k}\left(K ; \mathbb{R}^{d} \times \mathbb{R}_{\text {sym }}^{d \times d}\right)$ be the discontinuous finite element space of polynomial degree $k$. The discrete energy operator $M_{h}(t)=\sum_{K \in \mathcal{K}_{h}} M_{h, K}(t)$ for the discontinuous functions $\mathbf{v}_{h}=\sum_{K \in \mathcal{K}_{h}} \mathbf{v}_{h, K}$ and $\boldsymbol{\sigma}_{h}=\sum_{K \in \mathcal{K}_{h}} \boldsymbol{\sigma}_{h, K}$ is defined locally on $K$ by

$$
\left(M_{h, K}(t)\left(\mathbf{v}_{h, K}, \boldsymbol{\sigma}_{h, K}\right),\left(\mathbf{w}_{h, K}, \boldsymbol{\eta}_{h, K}\right)\right)_{0, K}=\left(\rho \mathbf{v}_{h, K}, \mathbf{w}_{h}\right)_{0, K}+\left(\mathbf{C}(t)^{-1} \boldsymbol{\sigma}_{h, K}, \boldsymbol{\eta}_{h}\right)_{0, K} .
$$

For the discontinuous functions, the derivatives are approximated by jump terms on the faces $\mathcal{F}=\bigcup_{K} \mathcal{F}_{K}$, where $\mathcal{F}_{K}$ are the faces on every element $K$. For inner faces $f \in \mathcal{F} \cap \Omega$, let $K_{f}$ be the neighboring cell such that $\bar{f}=\partial K \cap \partial K_{f}$. On boundary faces $f \in \mathcal{F} \cap \partial \Omega$ we set $K_{f}=K$. Let $\mathbf{n}_{K}$ be the outer unit normal vector on $\partial K$. We define the jump $\left[\mathbf{v}_{h}\right]_{K, f}=\mathbf{v}_{h, K_{f}}-\mathbf{v}_{h, K}$ on inner faces, where $\mathbf{v}_{h, K}$ denotes the continuous extension of $\left.\mathbf{v}_{h}\right|_{K}$ to $\bar{K}$. In the same way, the jump for the stress tensor is defined. On Dirichlet boundary faces, we set $\left[\mathbf{v}_{h}\right]_{K, f}=\mathbf{0}$ and $\left[\boldsymbol{\sigma}_{h}\right]_{K, f} \mathbf{n}=-2 \boldsymbol{\sigma}_{h} \mathbf{n}$. On Neumann boundaries, set $\left[\mathbf{v}_{h}\right]_{K, f}=-2 \mathbf{v}_{h}$ and $\left[\boldsymbol{\sigma}_{h}\right]_{K, f} \mathbf{n}=\mathbf{0}$.

The full upwind DG approximation $A_{h}(t)=\sum_{K \in \mathcal{K}_{h}} A_{h, K}(t)$ is defined by the local contributions

$$
\begin{aligned}
& \left(A_{h, K}(t)\left(\mathbf{v}_{h}, \boldsymbol{\sigma}_{h}\right),\left(\mathbf{w}_{h}, \boldsymbol{\eta}_{h}\right)\right)_{0, K}=\left(\operatorname{div} \boldsymbol{\sigma}_{h, K}, \boldsymbol{\psi}_{h, K}\right)_{0, K}+\left(\varepsilon\left(\mathbf{v}_{h, K}\right), \boldsymbol{\eta}_{h, K}\right)_{0, K} \\
& \quad+\frac{1}{2} \sum_{f \in \mathcal{F}_{K}}\left(\mathbf{n}_{K} \cdot\left(\left[\boldsymbol{\sigma}_{h, K}\right]_{K, f} \mathbf{n}_{K}+Z_{\mathrm{P}}(t)\left[\mathbf{v}_{h}\right]_{K, f}\right), \mathbf{n}_{K} \cdot\left(Z_{\mathrm{P}}(t)^{-1} \boldsymbol{\eta}_{h, K} \mathbf{n}_{K}+\mathbf{w}_{h, K}\right)\right)_{0, f} \\
& \quad+\frac{1}{2} \sum_{f \in \mathcal{F}_{K}}\left(\mathbf{n}_{K} \times\left(\left[\boldsymbol{\sigma}_{h, K}\right]_{K, f} \mathbf{n}_{K}+Z_{\mathrm{S}}(t)\left[\mathbf{v}_{h}\right]_{K, f}\right), \mathbf{n}_{K} \times\left(Z_{\mathrm{S}}(t)^{-1} \boldsymbol{\eta}_{h, K} \mathbf{n}_{K}+\mathbf{w}_{h, K}\right)\right)_{0, f}
\end{aligned}
$$


depending on the impedances $Z_{\mathrm{P}}(t)=\sqrt{\varrho(2 \mu(t)+\lambda(t))}$ and $Z_{\mathrm{S}}(t)=\sqrt{\varrho \mu(t)}$ of compressional waves and shear waves, respectively. Here, $(\cdot, \cdot)_{0, K}$ and $(\cdot, \cdot)_{0, f}$ denotes the $\mathrm{L}_{2}$ inner product in the elements and on the faces, respectively. The material parameters and thus the upwind flux and the operator $A_{h}$ depend on the material degradation (9) encoded in $\mathbf{C}(t)$. Note that the construction of the upwind flux can be extended to composite materials and discontinuous material parameters since it is computed by the exact solution of Riemann problems at interfaces. Here, the material tensor $\mathbf{C}(t)$ is continuous in space which simplifies the evaluation of the numerical flux on the element faces.

The boundary and volume data enter in the right-hand side $\mathbf{b}_{h}(t)=\sum_{K \in \mathcal{K}_{h}} \mathbf{b}_{h, K}(t)$ with

$$
\begin{aligned}
&\left(\mathbf{b}_{h, K}(t),\left(\mathbf{w}_{h, K}, \boldsymbol{\eta}_{h, K}\right)\right)_{0, K}=\left(\mathbf{f}(t), \mathbf{w}_{h, K}\right)_{0, K}+\sum_{f \in \mathcal{F}_{K} \cap \partial_{\mathrm{D}} \Omega}( \\
&+\left(\mathbf{n}_{K} \cdot \mathbf{v}_{\mathrm{D}}(t), \mathbf{n}_{K} \cdot\left(Z_{\mathrm{P}}(t)^{-1} \boldsymbol{\eta}_{h, K} \mathbf{n}_{K}+\mathbf{w}_{h, K}\right)\right)_{0, f} \\
&\left.+\left(\mathbf{n}_{K} \times \mathbf{v}_{\mathrm{D}}(t), \mathbf{n}_{K} \times\left(Z_{\mathrm{S}}(t)^{-1} \boldsymbol{\eta}_{h, K} \mathbf{n}_{K}+\mathbf{w}_{h, K}\right)\right)_{0, f}\right) \\
&+\sum_{f \in \mathcal{F}_{K} \cap \partial_{\mathrm{N}} \Omega}\left(\left(\mathbf{n}_{K} \cdot \mathbf{g}_{\mathrm{N}}(t), \mathbf{n}_{K} \cdot\left(\boldsymbol{\eta}_{h, K} \mathbf{n}_{K}+Z_{\mathrm{P}}(t) \mathbf{w}_{h, K}\right)\right)_{0, f}\right. \\
&\left.+\left(\mathbf{n}_{K} \times \mathbf{g}_{\mathrm{N}}(t), \mathbf{n}_{K} \times\left(\boldsymbol{\eta}_{h, K} \mathbf{n}_{K}+Z_{\mathrm{S}}(t) \mathbf{w}_{h, K}\right)\right)_{0, f}\right) .
\end{aligned}
$$

For the discretization in time, we distinguish two cases.

Elastic time step: If $\mathbf{C}\left(t_{n}\right)=\mathbf{C}\left(t_{n-1}\right)$ in the time step from $t_{n-1}$ to $t_{n}$, we use the implicit midpoint rule with time step size $\Delta t_{n}=t_{n}-t_{n-1}$. This corresponds for $\left(\mathbf{v}_{h}^{n}, \boldsymbol{\sigma}_{h}^{n}\right)$ to the equation

$$
\frac{1}{\Delta t_{n}} M_{h}^{n-1}\left(\begin{array}{c}
\mathbf{v}^{n}-\mathbf{v}^{n-1} \\
\boldsymbol{\sigma}^{n}-\boldsymbol{\sigma}^{n-1}
\end{array}\right)=\frac{1}{2} A_{h}^{n-1}\left(\begin{array}{c}
\mathbf{v}^{n}+\mathbf{v}^{n-1} \\
\boldsymbol{\sigma}^{n}+\boldsymbol{\sigma}^{n-1}
\end{array}\right)+\mathbf{b}_{h}^{n-1 / 2},
$$

with $M_{h}^{n-1}=M_{h}\left(t_{n-1}\right), A_{h}^{n-1}=A_{h}\left(t_{n-1}\right)$, and $\mathbf{b}_{h}^{n-1 / 2}=\mathbf{b}_{h}\left(\frac{1}{2}\left(t_{n}+t_{n-1}\right)\right)$, so that $\left(\mathbf{v}_{h}^{n}, \boldsymbol{\sigma}_{h}^{n}\right)$ is determined by solving the linear system

$$
\left(M_{h}^{n-1}-\frac{\Delta t_{n}}{2} A_{h}^{n-1}\right)\left(\begin{array}{l}
\mathbf{v}^{n} \\
\boldsymbol{\sigma}^{n}
\end{array}\right)=\left(M_{h}^{n-1}+\frac{\Delta t_{n}}{2} A_{h}^{n-1}\right)\left(\begin{array}{l}
\mathbf{v}^{n-1} \\
\boldsymbol{\sigma}^{n-1}
\end{array}\right)+\Delta t_{n} \mathbf{b}_{h}^{n-1 / 2} .
$$

This scheme is energy conserving for conforming solutions, so that in case of $\mathbf{b}_{h}^{n-1 / 2}=\mathbf{0}$ the mechanical energy is conserved $\mathcal{E}_{\mathrm{pf}}\left(t_{n}\right) \approx \mathcal{E}_{\mathrm{pf}}\left(t_{n-1}\right)$ up to the numerical dissipation induced by the nonconforming DG approximation.

Dissipative time step: If the material degrades in the time step, i.e., $\mathbf{C}\left(t_{n}\right) \neq \mathbf{C}\left(t_{n-1}\right)$, we use the implicit Euler method

$$
M_{h}^{n}\left(\begin{array}{c}
\mathbf{v}^{n} \\
\boldsymbol{\sigma}^{n}
\end{array}\right)=M_{h}^{n-1}\left(\begin{array}{c}
\mathbf{v}^{n-1} \\
\boldsymbol{\sigma}^{n-1}
\end{array}\right)+\Delta t_{n} A_{h}^{n}\left(\begin{array}{c}
\mathbf{v}^{n} \\
\boldsymbol{\sigma}^{n}
\end{array}\right)+\Delta t_{n} \mathbf{b}_{h}^{n}
$$

with $M_{h}^{n}=M_{h}\left(t_{n}\right), A_{h}^{n}=A_{h}\left(t_{n}\right)$, and $\mathbf{b}_{h}^{n}=\mathbf{b}_{h}\left(t_{n}\right)$, so that $\mathbf{y}_{h}^{n}$ is determined by the linear system

$$
\left(M_{h}^{n}-\Delta t_{n} A_{h}^{n}\right)\left(\begin{array}{c}
\mathbf{v}^{n} \\
\boldsymbol{\sigma}^{n}
\end{array}\right)=M_{h}^{n-1}\left(\begin{array}{c}
\mathbf{v}^{n-1} \\
\boldsymbol{\sigma}^{n-1}
\end{array}\right)+\Delta t_{n} \mathbf{b}_{h}^{n} .
$$

For $\mathbf{b}_{h}^{n}=\mathbf{0}$ the difference $\mathcal{E}_{\mathrm{pf}}\left(t_{n-1}\right)-\mathcal{E}_{\mathrm{pf}}\left(t_{n}\right)$ corresponds to the dissipated energy by opening the fracture zone so that the elastic domain gets smaller, i.e., $\Omega\left(s_{\text {inf }}\left(t_{n}\right)\right) \subset \Omega\left(s_{\text {inf }}\left(t_{n-1}\right)\right)$.

The linear systems (12) and (13) are well-defined, since the upwind discretization $-A_{h}(t)$ is positive semidefinite, so that the matrices $M_{h}^{n-1}-\frac{\Delta t_{n}}{2} A_{h}^{n-1}$ and $M_{h}^{n}-\Delta t_{n} A_{h}^{n}$ are regular. Moreover, both schemes are unconditional stable, also if the Courant-Friedrichs-Lewy condition $c_{\max } \Delta t_{n} \leq \frac{1}{2} h$ is not satisfied; herein, the maximal wave speed is denoted by $c_{\max }=\max \sqrt{(2 \mu+\lambda) / \varrho}$. On the other hand, $c_{\max } \Delta t_{n}=\mathcal{O}(h)$ is required for a balanced approximation error in space and time. Then we observe that the linear systems in (12) and (13) are well conditioned [Bohlen et al., 2020, Lem. 3.2], so that the approximate solution can be computed iteratively within a small number of steps, e.g., using a parallel GMRES method with block-Jacobi preconditioning. In the fracture zone where the material is degraded, the material stiffness and thus also the wave speed is reduced. In the limit this approximates Neumann boundary conditions at crack interfaces. 


\section{Computational Dynamic fracture}

We combine the DG discretization for the hyperbolic linear wave equation in the discontinuous finite element space $V_{h}^{\mathrm{dg}} \subset \mathrm{L}_{2}\left(\Omega ; \mathbb{R}^{d} \times \mathbb{R}_{\mathrm{sym}}^{d \times d}\right)$ with a parabolic phase-field approximation by lowest order conforming finite elements $V_{h}^{\text {cf }} \subset \prod \mathbb{P}(K) \cap \mathrm{C}(\bar{\Omega}) \subset \mathrm{H}^{1}(\Omega)$. Then, $\phi_{h} \in V_{h}^{\text {cf }}$ is uniquely defined by the nodal values $\left(\phi_{h}(\mathbf{x})\right)_{\mathbf{x} \in \mathcal{C}_{h}}$, where $\mathcal{C}_{h} \subset \bar{\Omega}$ are the vertices of the elements $K \in \mathcal{K}_{h}$. Since the finite element approximation of the phase field is continuous, it allows a continuous evaluation of the degraded material parameters on the element faces within the computation of the numerical flux in the wave discretization.

We use a staggered time-discrete scheme, updating alternately the elastic system $\left(\mathbf{v}_{h}^{n}, \boldsymbol{\sigma}_{h}^{n}\right)$ and the phase field $s_{h}^{n}$, and depending on the phase-field evolution the material is degraded only in time steps where the elastic domain (determined by $s_{\min }$ ) becomes smaller. The main steps of the algorithm are summarized in Fig. 1.

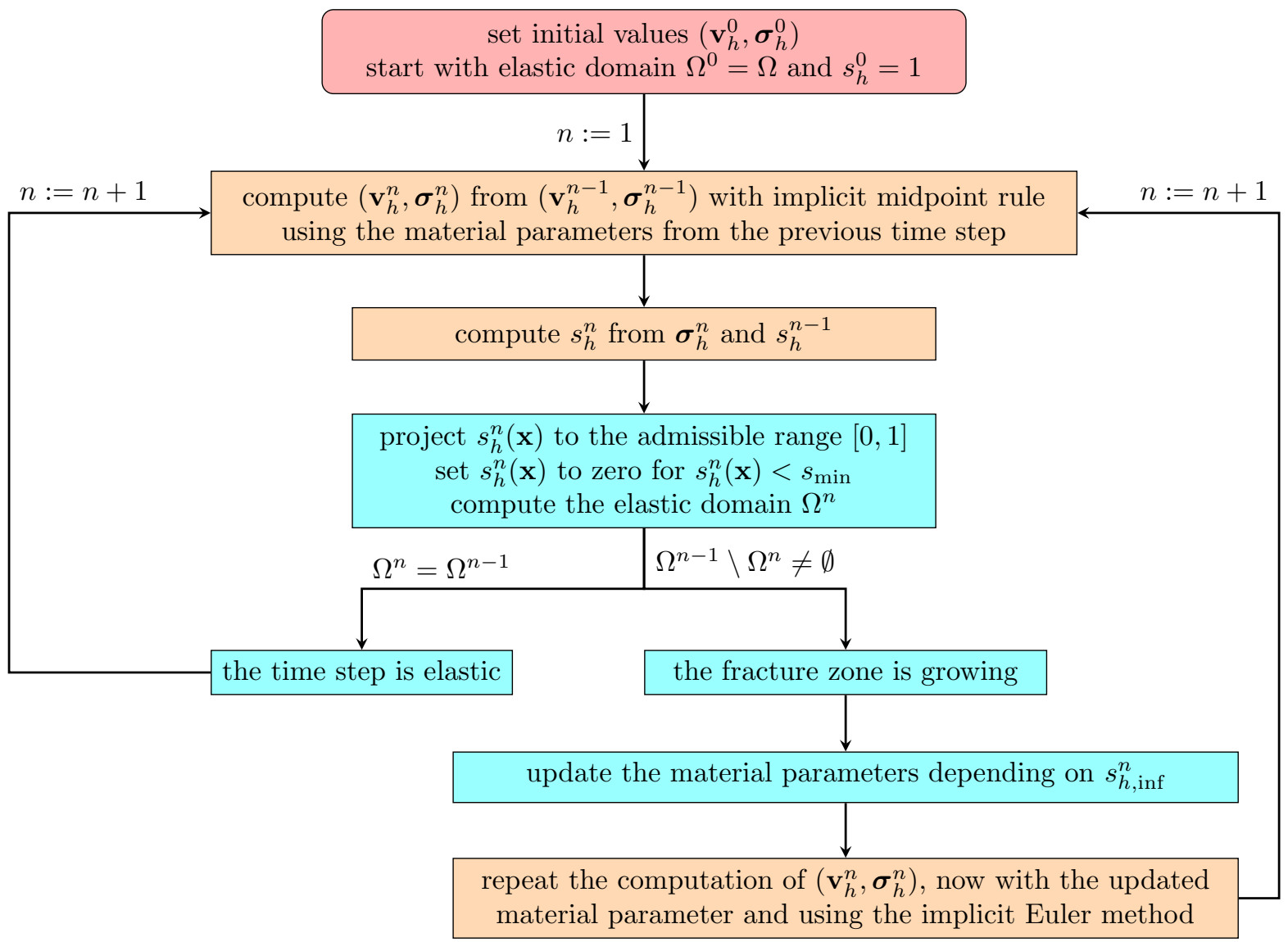

FIgURE 1. The staggered scheme for the elastic variables $\left(\mathbf{v}_{h}^{n}, \boldsymbol{\sigma}_{h}^{n}\right)$ and the phase field $s_{h}^{n}$. 
We start with initial values $\left(\mathbf{v}_{h}^{0}, \boldsymbol{\sigma}_{h}^{0}, s_{h}^{0}\right) \in V_{h}^{\mathrm{dg}} \times V_{h}^{\mathrm{cf}}$ in the material without fracture, i.e., we set $\Omega^{0}=\Omega$, $s_{h}^{0}=s_{h \text {,inf }}^{0}=1$, and $\mathbf{C}^{0}=\mathbf{C}$. For elasticity we set $\mathbf{v}_{h}^{0}=\Pi_{h} \mathbf{v}_{0}$ and $\boldsymbol{\sigma}_{h}^{0}=\Pi_{h} \boldsymbol{\sigma}_{0}$ with a suitable projection or interpolation $\Pi_{h}$. Furthermore, we select time steps $0<\Delta t_{\mathrm{pf}} \leq \Delta t_{\mathrm{el}}$ for the evolution of the phase-field and the elastic wave propagation. In addition, the displacement can be approximated by integrating the velocity; we set $\mathbf{u}_{h}^{0}=\Pi_{h} \mathbf{u}_{0}$. We start with $\Delta t_{1}=\Delta t_{\mathrm{el}}$.

In every time step $n=1,2,3, \ldots$ we proceed as follows:

(S1) We compute from $\left(\mathbf{v}_{h}^{n-1}, \boldsymbol{\sigma}_{h}^{n-1}\right)$ the solution for the next time step $\left(\mathbf{v}_{h}^{n}, \boldsymbol{\sigma}_{h}^{n}\right) \in V_{h}^{\mathrm{dg}}$ with the implicit midpoint rule (12), i.e.,

$$
\begin{aligned}
& \left(\left(M_{h}^{n-1}-\frac{\Delta t_{n}}{2} A_{h}^{n-1}\right)\left(\mathbf{v}_{h}^{n}, \boldsymbol{\sigma}_{h}^{n}\right),\left(\mathbf{w}_{h}, \boldsymbol{\eta}_{h}\right)\right)_{0, \Omega} \\
& \quad=\left(\left(M_{h}^{n-1}+\frac{\Delta t_{n}}{2} A_{h}^{n-1}\right)\left(\mathbf{v}_{h}^{n-1}, \boldsymbol{\sigma}_{h}^{n-1}\right),\left(\mathbf{w}_{h}, \boldsymbol{\eta}_{h}\right)\right)_{0, \Omega}+\Delta t_{n}\left(\mathbf{b}_{h}^{n-1 / 2},\left(\mathbf{w}_{h}, \boldsymbol{\eta}_{h}\right)\right)_{0, \Omega}, \quad\left(\mathbf{w}_{h}, \boldsymbol{\eta}_{h}\right) \in V_{h}^{\mathrm{dg}} .
\end{aligned}
$$

Here, $\left(\mathbf{v}_{h}^{n}, \boldsymbol{\sigma}_{h}^{n}\right)$ is just a candidate for the next time step which will be accepted only if the fracture criterion in step (S3) shows that this step is elastic; otherwise, $\left(\mathbf{v}_{h}^{n}, \boldsymbol{\sigma}_{h}^{n}\right)$ will be recomputed in (S6).

(S2) Depending on $\boldsymbol{\sigma}_{h}^{n}$, we approximate the phase field $s_{h}^{n} \in V_{h}^{\text {cf }}$ by the implicit Euler method applied to (8), i.e., by solving

$$
\left(\tau_{\mathrm{r}} s_{h}^{n}, \phi_{h}\right)_{0, \Omega}+\Delta t_{n} M_{\text {geom }}\left(\left(\left(s_{h}^{n}-1\right), \phi_{h}\right)_{0, \Omega}+\left(l_{\mathrm{c}}^{2} \nabla s_{h}^{n}, \nabla \phi_{h}\right)_{0, \Omega}\right)=\left(\tau_{\mathrm{r}} s_{h}^{n-1}-\Delta t_{n} Y_{\mathrm{el}}\left(\boldsymbol{\sigma}_{h}^{n}\right), \phi_{h}\right)_{0, \Omega}, \quad \phi_{h} \in V_{h}^{\mathrm{cf}} .
$$

Again we note that $s_{h}^{n}$ is just a candidate since it will be modified in the next step (S3).

(S3) On all nodal points $\mathbf{x} \in \mathcal{C}_{h}$, the phase field $s_{h}^{n}(\mathbf{x})$ is projected to $[0,1]$ by

$$
s_{h}^{n}(\mathbf{x}):= \begin{cases}1 & s_{h}^{n}(\mathbf{x}) \geq 1 \\ s_{h}^{n}(\mathbf{x}) & s_{\min } \leq s_{h}^{n}(\mathbf{x})<1 \\ 0 & s_{h}^{n}(\mathbf{x})<s_{\min } \text { or } s^{n-1}(\mathbf{x})=0\end{cases}
$$

and we set

$$
s_{h, \text { inf }}^{n}(\mathbf{x})=\min \left\{s_{h, \text { inf }}^{n-1}(\mathbf{x}), s_{h}^{n}(\mathbf{x})\right\}, \quad \Omega^{n}=\left\{\mathbf{x} \in \Omega^{n-1}: s_{h, \text { inf }}^{n}(\mathbf{x}) \geq s_{\min }\right\} .
$$

(S4) If $\Omega^{n}=\Omega^{n-1}$, the time step is elastic and $\mathbf{C}^{n}=\mathbf{C}^{n-1}$; then, we accept the elastic solution $\left(\mathbf{v}_{h}^{n}, \boldsymbol{\sigma}_{h}^{n}\right)$ and directly proceed with (S7).

(S5) Otherwise, if the fracture zone is growing by $\Omega^{n-1} \backslash \Omega^{n} \neq \emptyset$, we update the material, i.e.,

$$
\mathbf{C}^{n}(\mathbf{x})=s_{\text {inf }}^{n}(\mathbf{x}) \mathbf{C}+\left(1-s_{\text {inf }}^{n}(\mathbf{x})\right) \mathbf{C}_{\text {reg }} .
$$

(S6) We repeat the computation of $\left(\mathbf{v}^{n}, \boldsymbol{\sigma}^{n}\right)$ with the updated material:

Using the implicit Euler method (13), we compute $\left(\mathbf{v}_{h}^{n}, \boldsymbol{\sigma}_{h}^{n}\right) \in V_{h}^{\mathrm{dg}}$ by solving

$$
\begin{aligned}
&\left(\left(M_{h}^{n}-\right.\right.\left.\left.\Delta t_{n} A_{h}^{n}\right)\left(\mathbf{v}_{h}^{n}, \boldsymbol{\sigma}_{h}^{n}\right),\left(\mathbf{w}_{h}, \boldsymbol{\eta}_{h}\right)\right)_{0, \Omega} \\
& \quad=\left(M_{h}^{n-1}\left(\mathbf{v}_{h}^{n-1}, \boldsymbol{\sigma}_{h}^{n-1}\right),\left(\mathbf{w}_{h}, \boldsymbol{\eta}_{h}\right)\right)_{0, \Omega}+\Delta t_{n}\left(\mathbf{b}_{h}^{n},\left(\mathbf{w}_{h}, \boldsymbol{\eta}_{h}\right)\right)_{0, \Omega}, \quad\left(\mathbf{w}_{h}, \boldsymbol{\eta}_{h}\right) \in V_{h}^{\mathrm{dg}} .
\end{aligned}
$$

(S7) We set $\mathbf{u}_{h}^{n}=\mathbf{u}_{h}^{n-1}+\Delta t_{n} \mathbf{v}_{h}^{n}$.

If $s_{h}^{n}=s_{h}^{n-1}$, we expect that the next time step will also be elastic and we set $\Delta t_{n+1}=\Delta t_{\mathrm{el}}$; otherwise, we set $\Delta t_{n+1}=\Delta t_{\mathrm{pf}}$. Then, we continue with the next time step $n:=n+1$ and we proceed with (S1). 


\section{NumericAl EXPERIMENTS}

The numerical versatility of our staggered algorithm is illustrated with examples in one, two and three dimensions.

\subsection{Simulation of a Hopkinson bar experiment}

We start with the simulation of a spalling experiment performed in our lab. In these experiments, the setup of a Hopkinson-Pressure bar is modified so that the specimen is placed at the end of a long incident bar that a striker hits. The induced pressure pulse is transmitted via the incident bar into the specimen and reflected at its free end. The resulting tensional wave determines an inhomogeneous stress state, and when the tensile stress exceeds the material's strength, the specimen fractures, see Fig. 2. The stress value at fracture is considered to be the dynamic tensile resistance of the material, cf. [Weinberg and Khosravani, 2018]. A successful experiment requires specimens with a certain tension-compression asymmetry; we investigated Ultra-High Performance Concrete with $E=50 \mathrm{GPa}$ and $\sigma_{c}=18 \mathrm{MPa}$, cf. [Khosravani et al., 2019].
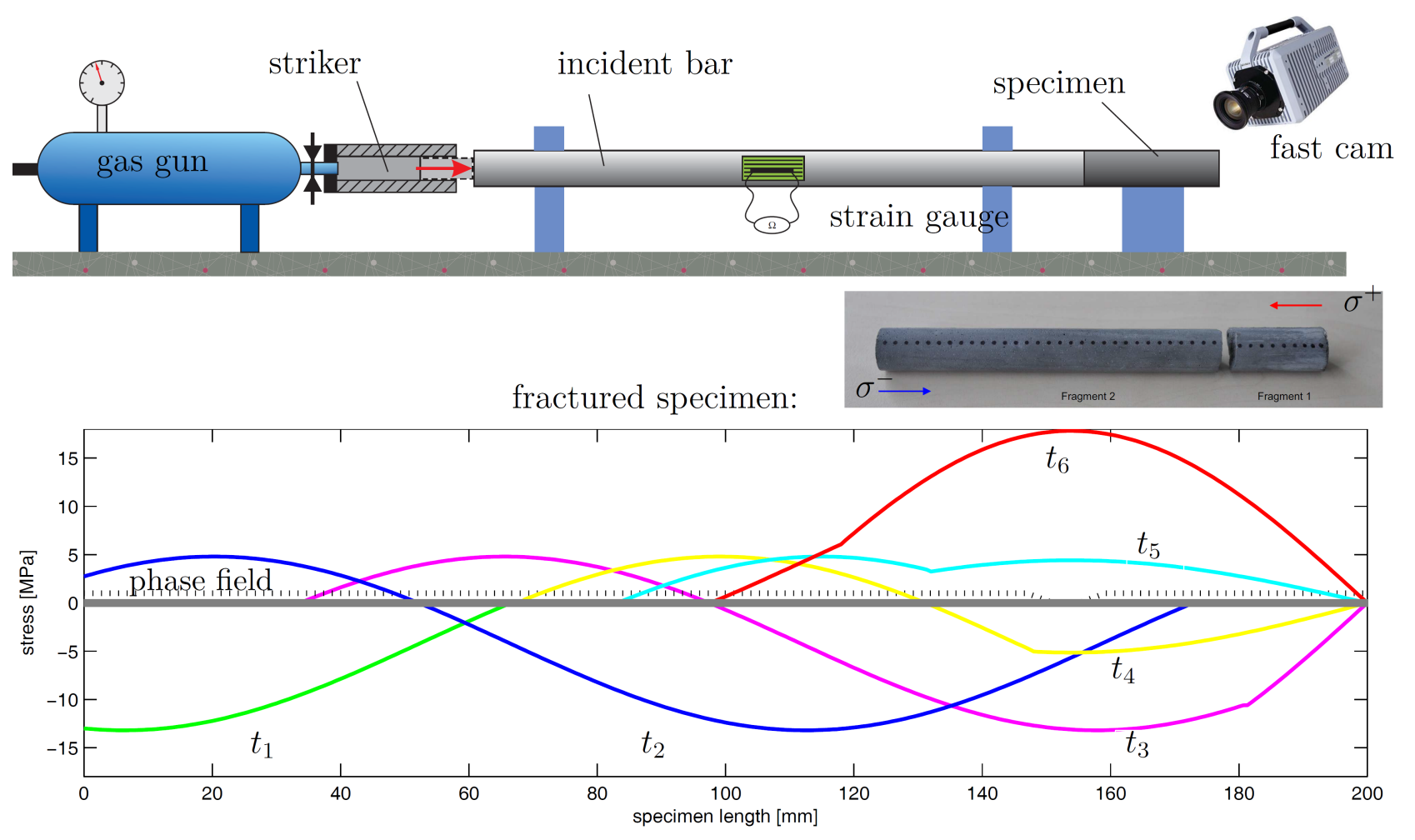

FIGURE 2. Hopkinson bar spallation experiment with a fractured specimen and simulation of the stress wave for different times; $t_{1}$ : incoming pressure wave pulse, $t_{2}$ : traveling wave, $t_{3}-t_{4}$ : superposition during reflection at the free end, and $t_{5}$ : the peak stress which causes spallation; the dashed line is the phase field.

The evaluation of the experiment is based on the one-dimensional linear wave theory and our DG simulation of this setting completely reproduces the theoretical results. For some instances of time, the waves in the specimen are displayed in Fig. 2 whereby the analytical and the numerical curve basically lay on top of each other. The phase-field fracture approximation shows a crack at the critical stress of $18 \mathrm{MPa}$.

Clearly, this setting is too simple to illustrate the advantages of our new numerical approach. Therefore we modified it in such a way that the examples show waves propagation and superposition in two or three dimensions. To avoid effects which are only caused by symmetry, we choose a non-uniformly curved geometry with non-symmetric wave pulses from left and right. The pulses travel with the sample's wave speed, superpose, are then reflected at the free boundary, and continue traveling with inverted amplitude until they induce cracks. In the following, geometry and all material parameters are dimensionless but the values of choice (if understand in units $\mathrm{N}, \mathrm{mm}$ and $\mu \mathrm{s}$ ) correspond to a typical hard plastic such as polymethyl methacrylate (PMMA). 


\subsection{A 2D curved bar}

For plane strain computations a unit reference domain is mapped into a curved configuration $\Omega=\varphi\left(\Omega_{\mathrm{ref}}\right)$ with

$$
\Omega_{\mathrm{ref}}=(-0.5,0.5) \times(0.03125,0.03125) \text { and } \varphi\left(x_{1}, x_{2}\right)=\left(x_{1}, \cos \left(0.5 x_{1} \pi\right)\right)+x_{2}\left(\sin \left(0.5 x_{1} \pi\right), \cos \left(0.5 x_{1} \pi\right)\right) .
$$

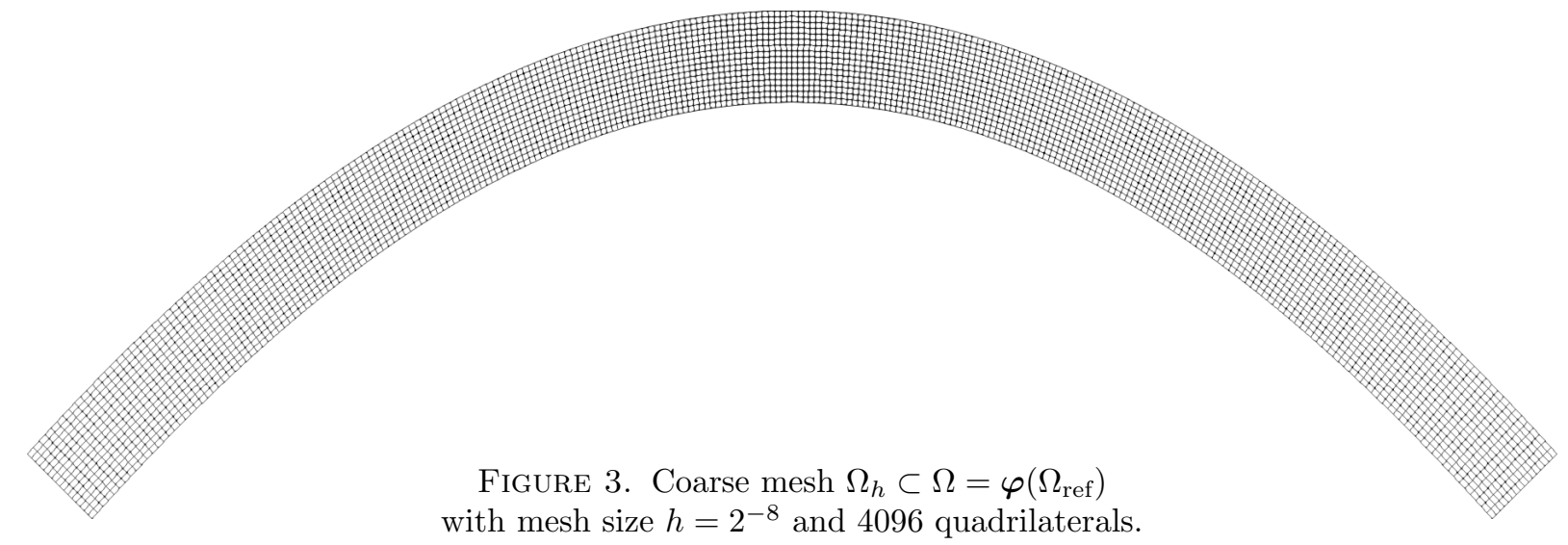

The material is isotropic and linear elastic with $\mathbf{C} \varepsilon=2 \mu \varepsilon+\lambda \operatorname{trace}(\varepsilon)$ id, $\mu=1, \lambda=2$. With density $\rho=1$ this gives the speed $c_{\mathrm{P}}=2$ for compressional waves and $c_{\mathrm{S}}=1$ for shear waves.

For the phase-field model, we use $\sigma_{\mathrm{c}}=27, M_{\text {geom }}=0.01, l_{\mathrm{c}}=0.0005, s_{\min }=0.01$, and for the material degradation $\mathbf{C}_{\text {reg }} \varepsilon=2 \mu_{\text {reg }} \varepsilon+\lambda_{\text {reg }}$ trace $(\varepsilon)$ id with $\mu_{\text {reg }}=10^{-7} \mu$ and $\lambda_{\text {reg }}=10^{-7} \lambda$. The phase-field is approximated with bilinear conforming finite elements; the mesh is illustrated in Fig. 3 for the coarsest level with mesh size $h=2^{-8}$ and $16 \cdot 256$ quadrilaterals, and uniformly refined up to $h=2^{-11}$ with $128 \cdot 2048$ quadrilaterals. Velocity and stress are approximated with discontinuous bilinear finite elements.

The model is loaded by a smooth pressure pulse $\boldsymbol{\sigma} \mathbf{n}=g_{\mathrm{N}}(t) \mathbf{n}$ at the left and right boundary with

$$
g_{\mathrm{N}}\left(t, \varphi\left( \pm 0.5, x_{2}\right)\right)=a_{ \pm}\left(c_{\mathrm{P}} t-S_{ \pm}\right), \quad a_{ \pm}(s)=A_{ \pm} \exp \left(\frac{-1}{w_{ \pm}^{2}-s^{2}}\right), \quad t \in\left(0, t_{\text {init }}\right) .
$$

The impulse width is $w_{ \pm}=0.3$ and the duration is $t_{\text {init }}=0.24$. This corresponds to incoming compressional waves from left and right. To break symmetry the impulse on the right side is $5 \%$ stronger, i.e., we set for the amplitude $A_{+}=1.05 A_{-}$and use the time shift parameters $S_{+}=1.25$ and $S_{-}=-1.03$. In that way a crack will be initiated by the superposition of the traveling waves close to the center at $x_{1}=0$ but not exactly aligned with the mesh. On the remaining boundaries and for $t \geq t_{\text {init }}$ we use homogeneous Neumann boundary conditions $\mathbf{g}_{\mathrm{N}}=0$.

In this numerical experiment, the following behavior is observed (illustrated by snapshots of the solution in Fig. 4): Compressional waves are initiated for $t \in[0,0.24]$ by a pressure impulse from left and right. Then, they travel, and for $t \in[0.75,1]$ the waves are reflected at the free boundaries and result in tensional waves. At $t=1.2$, by superposition of the reflected waves, the tension increases, and the traction forces become so large that the driving force $Y_{\text {el }}$ gets positive and the fracture criterion is met; the phase field evolves and the material breaks. Since the domain is curved, the stress is larger at the top side and the crack grows from top to bottom. For $t>1.2$, the waves are reflected at the crack interfaces and become compressive. Then, for $t \in[1.6,1.65]$ the waves are reflected again at the free boundaries and turn into tension. Finally, for $t=[1.65,1.71]$ by superposition of incoming and reflected waves, a second fracture zone is initiated. Because of the non-symmetric setting, this secondary crack is slightly different at the left and the right hand side. 


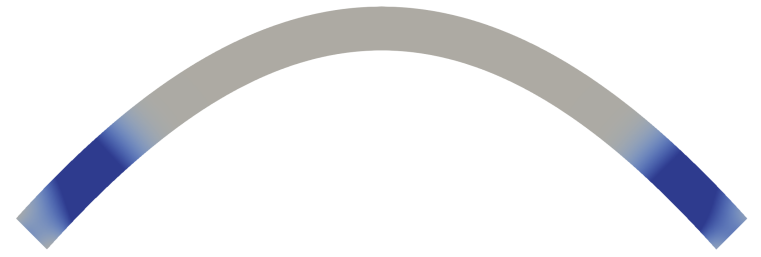

$t=0.18$
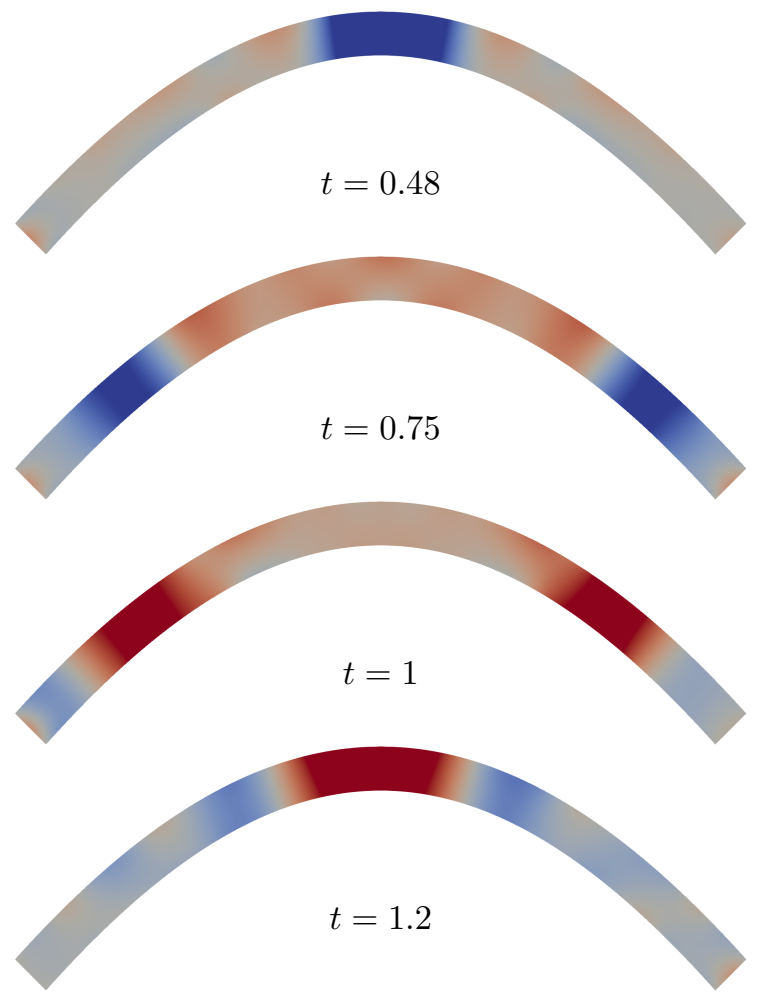
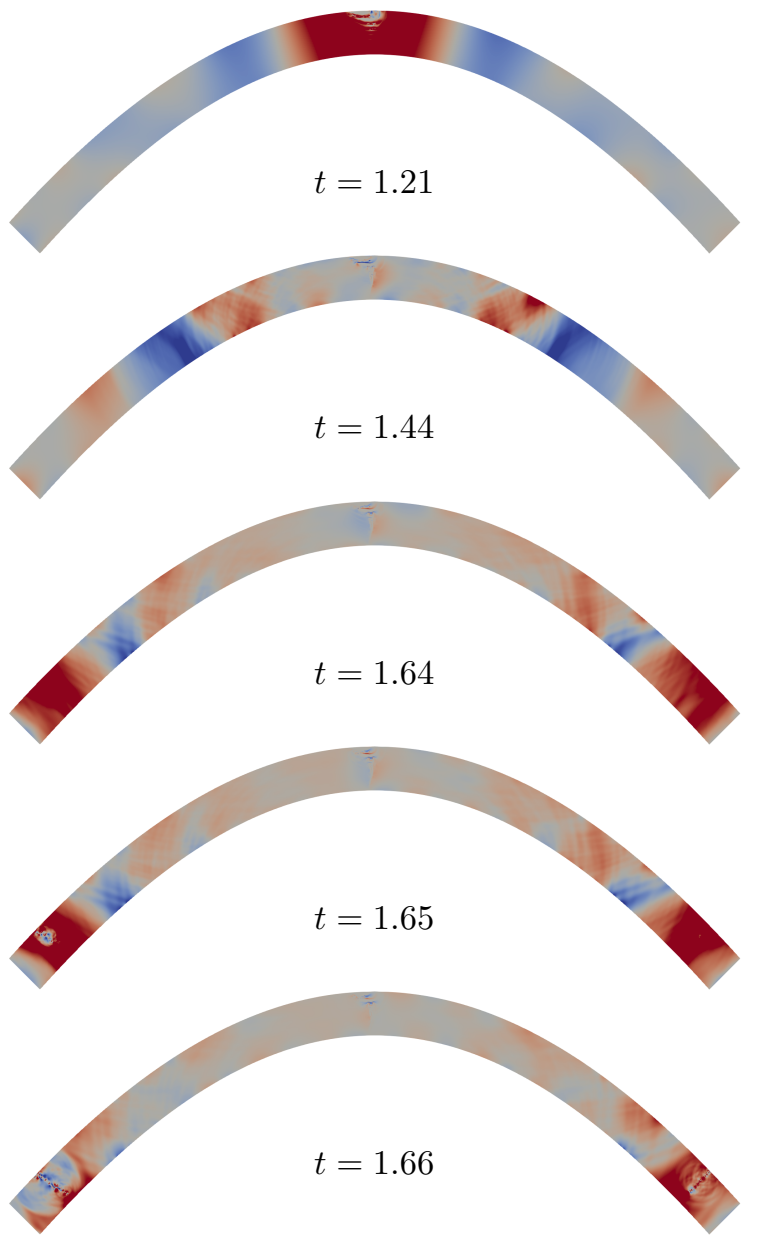

Figure 4. Traveling pressure wave, $p=\operatorname{tr} \sigma$ for $t \in[0,2]$, for the plane curved bar; red - compression, blue - tension.

The resulting phase-field approximation of the fracture evolution is shown in Fig. 5. We use a fixed length scale of $l_{\mathrm{c}}=0.0005$ which determines the fracture regularization by the phase-field approach. This requires to use a mesh size of $h=2^{-8}$ to resolve the main features of the fracture pattern including primary and the secondary cracks. Then, using finer meshes, we observe than the overall setting is converging, even though the cracks are more complex on finer meshes. The accuracy of the computation is limited by the a priori choice of the phase-field regularization given by the length scale parameter, resulting in a diffusive approximation of the crack. Moreover, we observe that larger values of the scaling parameter $M_{\text {geom }}$ or of the length scale $l_{\mathrm{c}}$ increase the dissipation and so, after the primary crack, the remaining mechanical energy is not sufficient for the initiation of further cracks.

In this numerical experiment we use on level $m=8,9,10,11$ the mesh size $h=2^{-m}$ with $2^{m+4}$ quadrilateral elements and 45 degrees of freedom per element for the velocity and stress approximation. The smallest computation on level $m=8$ with 2000 time steps runs on a laptop within 14 hours, so that we need about 25 seconds per time step. The finer computations were realized on a parallel computing cluster with different numbers of cores.

In Fig. 6 the evolution of the secondary crack at the right hand side of the model is displayed on the finest mesh. Here it can be observed that the crack propagates with approximately $50 \%$ of the wave speed. Such cracks, with a velocity of about the Rayleigh-wave speed at the crack tip, are typical for dynamic brittle fracture, cf. [Freund, 1998, Chap. 7.4]. 


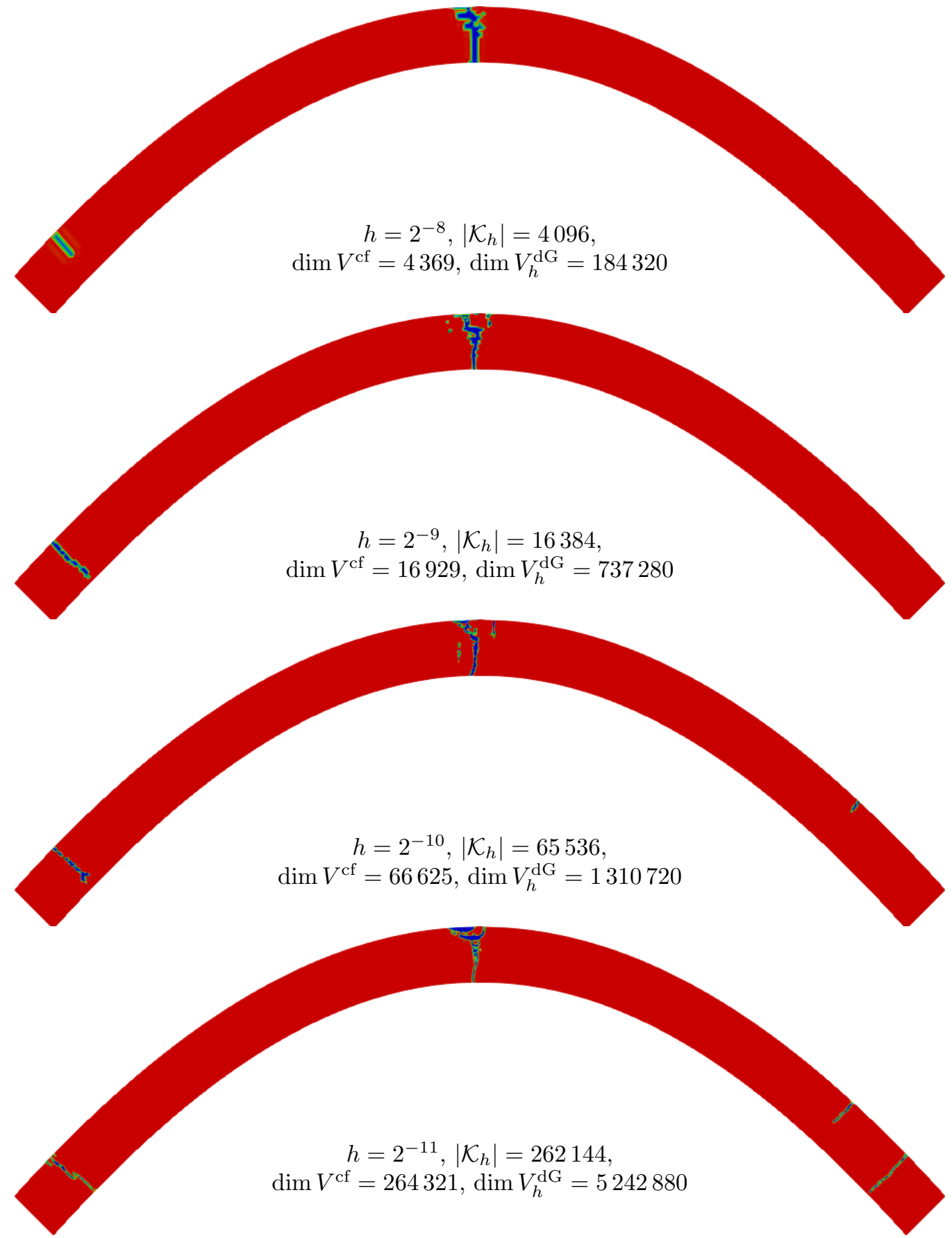

FiguRE 5. Phase-field fracture approximation $s_{h \text {,inf }}$ at $t=2$ computed with time step sizes $\Delta t_{\mathrm{el}}=0.001$ and $\Delta t_{\mathrm{pf}}=0.0005$ on different meshes with mesh size $h$, number of elements $\left|\mathcal{K}_{h}\right|$, dimension of the finite element spaces for the phase field $\operatorname{dim} V^{\mathrm{cf}}$ and the wave system $\operatorname{dim} V_{h}^{\mathrm{dG}}$.

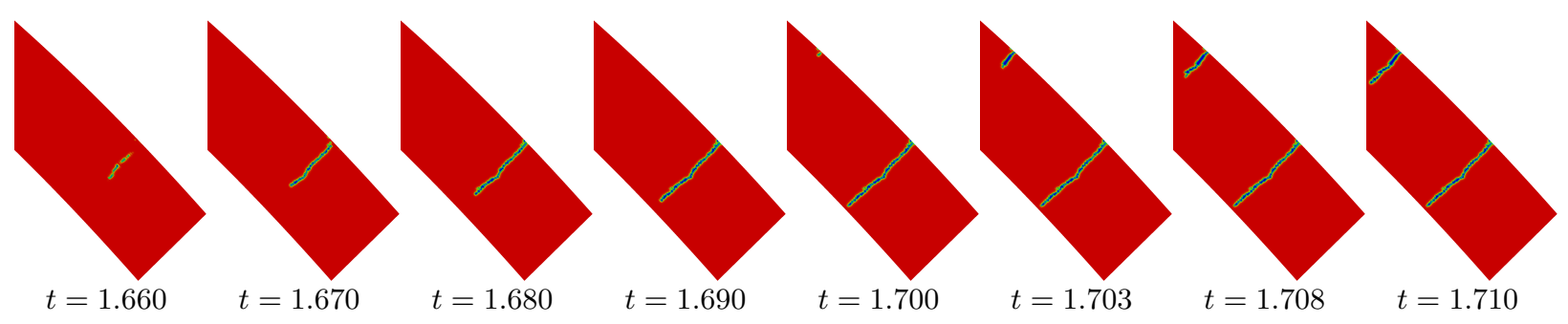

FiguRE 6. Evolution of a secondary crack close to the right boundary, $s_{h, \text { inf }}$ for $t \in[1.66,1.71]$; the crack tip propagates with approximately $50 \%$ of the wave speed. 


\subsection{A 3D experiment}

Here we show that the observed qualitative behavior transfers to three space dimensions. The configuration is now somewhat simplified, i.e., the curved bar-like domain is shorter, and the pressure impulse is modified so that already the first superposition of the waves generates tension. At the front side of the bar, the crack criterion is met first. The wave propagation and the evolution of cracks patterns at the surface are illustrated in Fig. 7.

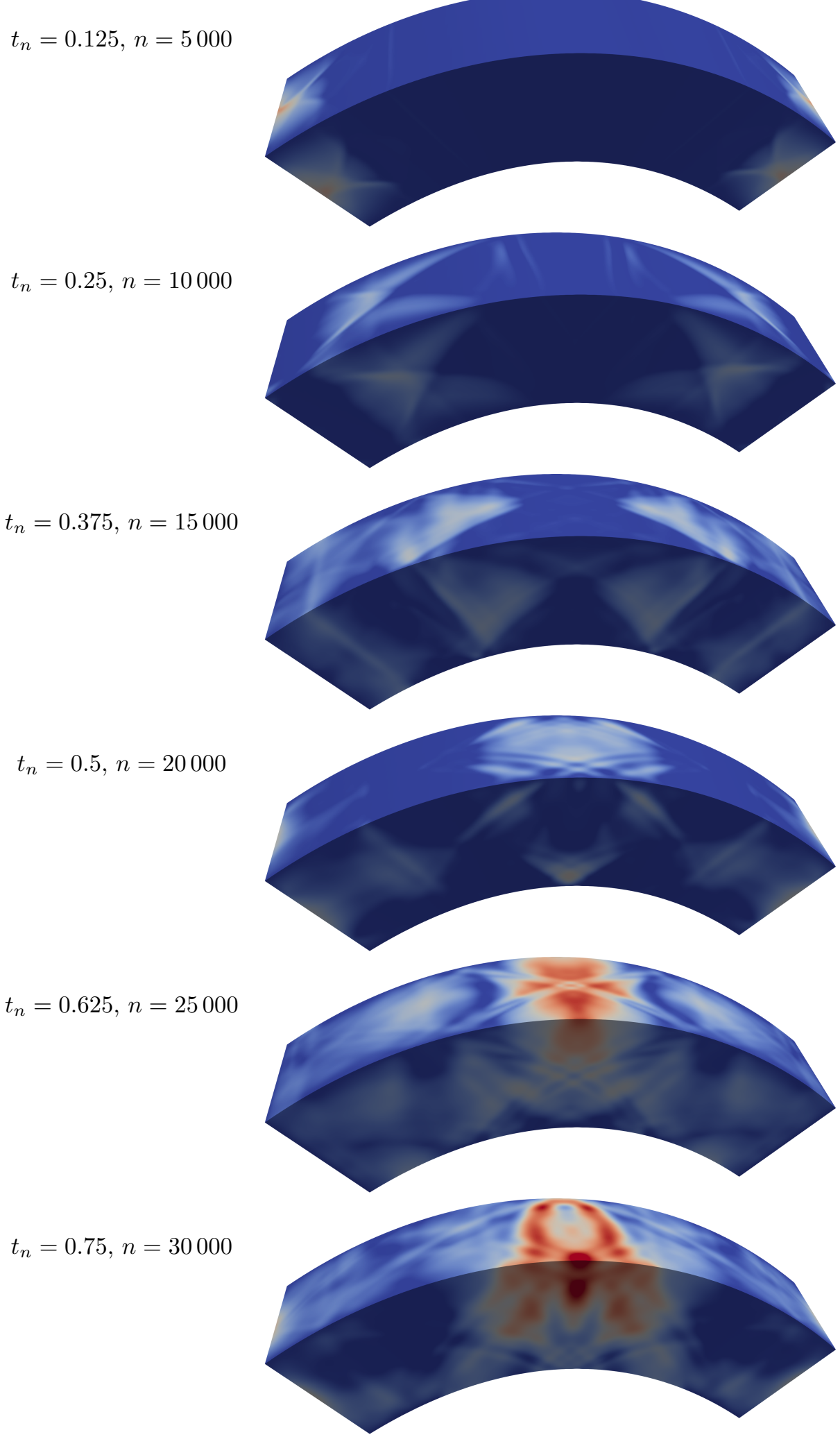

FiguRE 7. Principle stress evolution $\sigma_{\text {I }}$ for the 3D configuration on 1048576 hexahedra. 
In this simulation we use a fixed time step size $\Delta t_{\mathrm{el}}=\Delta t_{\mathrm{pf}}=0.000025$, a trilinear DG finite element space for the elastic system with 75497472 degrees of freedom, and a phase-field approximation with 1085825 degrees of freedom on a hexahedral mesh. The full simulation requires approx. 20 hours on 2048 parallel computing cores on HoreKA (https://www.nhr.kit.edu/userdocs/horeka), so that per time step we need only a few seconds for the parallel preconditioned GMRES solver.

$$
t_{n}=0.75, n=30000
$$

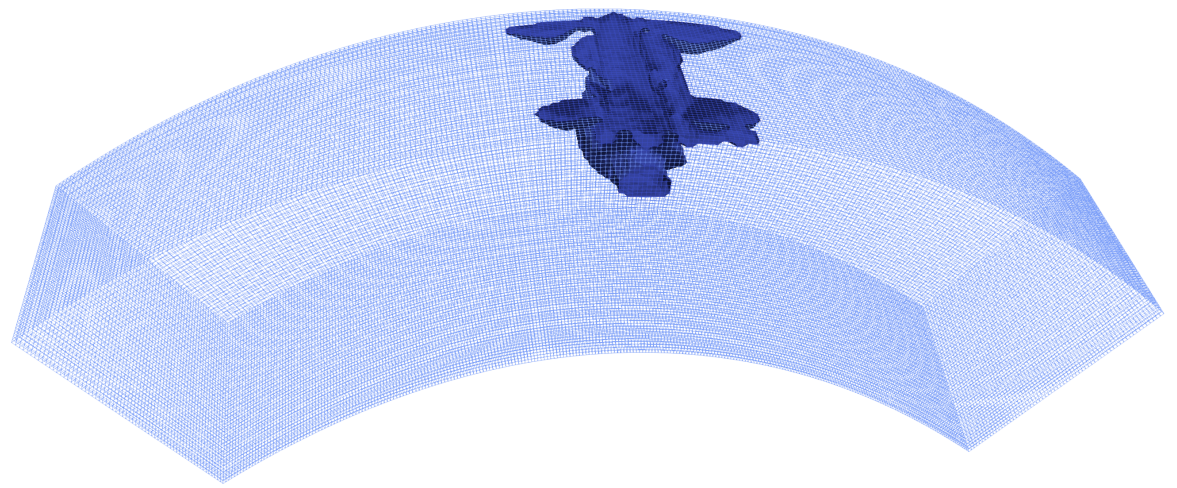

FIGURE 8. Phase-field approximation $s_{h, \text { inf }}$ at final time $t=0.75$.

The resulting phase-field approximation at the final time $t_{n}=0.75$ is shown in Fig. 8 , and the evolution of the phase-field approximation at different time steps is illustrated in Fig. 9. Comparing the numerical approximation of the phase field on different meshes, we observe that the crack evolution can be described on the coarser level, but more details are included in the finer resolution. On coarser levels, the complexity of the crack pattern cannot be resolved, so that indeed such detailed computations are required to obtain fine fragment spallation.

phase-field approximation with 1085825 DoFs on 1048576 hexahedra

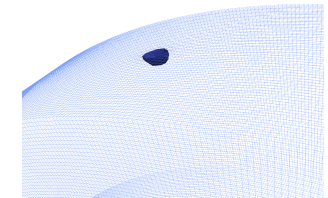

$t=0.65$

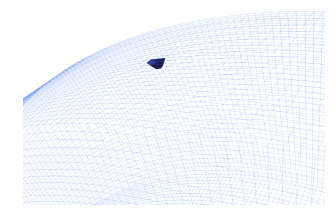

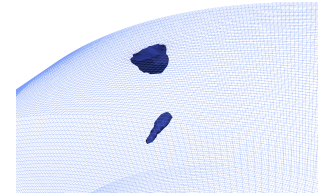

$t=0.6575$

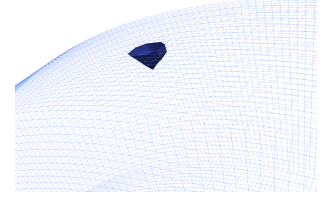

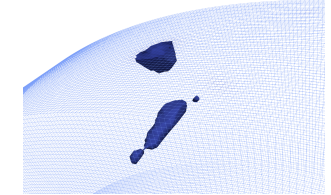

$t=0.66$

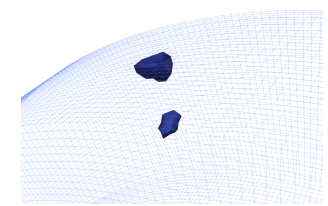

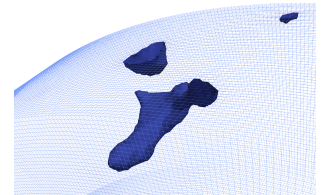

$t=0.665$

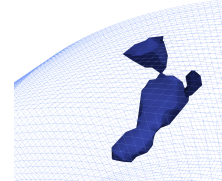

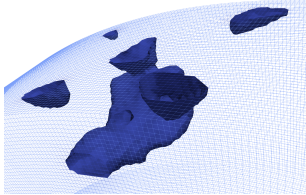

$t=0.68$

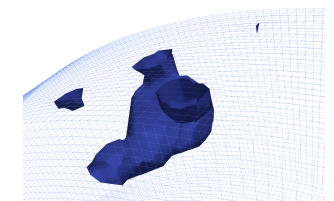

phase-field approximation with 140481 DoFs on 131072 hexahedra

Figure 9. Details of the phase-field evolution at different times with different mesh resolution.

Again, we observe wave propagation and crack pattern interaction as a purely dynamic phenomenon that is only driven by the stress interaction and does not require any geometric initiation. Our new first-order method for wave propagation is clearly able to recover spallation phenomena.

An overview of the software design is reported in [Baumgarten and Wieners, 2021], and the code is available on https://git.scc.kit.edu/mpp/dgwave/-/tree/fracture1.1 including the configuration for the presented examples.

\section{Conclusion And future Perspectives}

In this contribution, we aim to establish a new algorithmic methodology for wave propagation with dynamic fracture. This methodology allows to reliably predict fracture and fragmentation due to traveling waves and their superposition as well as the wave reflections at the fracture interfaces.

The major difference to established methods in [Miehe et al., 2010, Borden et al., 2012] is the formulation and approximation of wave propagation as a first-order hyperbolic system for velocity and stress, using techniques that are well established in fluid dynamics. It remains an open question whether it is possible to show the presented fracture dynamic with conforming finite element approximations of the displacements, particularly 
since DG methods can propagate discontinuities initiated by crack opening. Further investigations are required to see if such a fracture dynamic can also be approximated with a Newmark-type approach.

We focus here on the basic formulation for linear elastic materials; this will be extended to more general material classes and crack-driving forces in a next step.

In our examples we employ a crack-driving force based on the maximum principal stress $Y_{\mathrm{el}}=\max \left\{\frac{\sigma_{\mathrm{I}}}{\sigma_{\mathrm{c}}}-1,0\right\}$.

The corresponding state of tension defines the (reversible) phase-field evolution

$$
0 \in \tau_{\mathrm{r}} \dot{s}+Y_{\mathrm{el}}-M_{\mathrm{geom}} \partial \gamma_{\mathrm{c}}(s)+\partial \chi_{[0,1]}(s),
$$

and transfers to the irreversible phase-field fracture evolution

$$
0 \in \tau_{\mathrm{r}} \dot{s}+\partial \chi_{(-\infty, 0)}(\dot{s})+Y_{\text {el }}-M_{\text {geom }} \partial \gamma_{\mathrm{c}}(s) .
$$

The maximum principal stress driving force is a common fracture criterion and a straightforward choice for brittle materials. Likewise, the usual variational formulation of the phase-field driving force could be employed. This formulation requires the split of the energy density into a tensile and a compressive energy functional $W_{\mathrm{pf}}(\varepsilon, s)$, so that the stress response and phase-field driving force are conjugate and defined by

$$
\boldsymbol{\sigma}=\partial_{\varepsilon} W_{\mathrm{pf}}(\varepsilon, s), \quad Y_{\mathrm{el}}=-\partial_{s} W_{\mathrm{pf}}(\varepsilon, s) .
$$

The phase-field fracture formulation can then be extended to problems with finite deformations, see [Hesch et al., 2017, Weinberg et al., 2016, Thomas et al., 2020]. However, it remains an open question how to construct the corresponding discontinuous Galerkin framework. Another topic of ongoing research is the extension of our scheme to viscoelastic media, as it is analyzed in [Thomas and Tornquist, 2021] and formulated in a phase-field setting in [Bartels et al., 2020], since wave propagation always is dispersive in natural media.

Acknowledgement. The authors gratefully acknowledge the support of the Deutsche Forschungsgemeinschaft (DFG) within the Priority Program 2256 "Variational Methods for Predicting Complex Phenomena in Engineering Structures and Materials" in the projects WE 2525/15-1 and WI 1430/9-1.

\section{REFERENCES}

[Ambati and Lorenzis, 2016] Ambati, M. and Lorenzis, L. D. (2016). Phase-field modeling of brittle and ductile fracture in shells with isogeometric nurbs-based solid-shell elements. Computer Methods in Applied Mechanics and Engineering (CMAME).

[Anderson, 2004] Anderson, T. (2004). Fracture Mechanics: Fundamentals and Applications. CRC Press, 3rd edition.

[Bartels et al., 2020] Bartels, S., Milicevic, M., Thomas, M., Tornquist, S., and Weber, N. (2020). Approximation schemes for materials with discontinuities. http://www.wias-berlin.de/preprint/2799/wias_preprints_2799.pdf.

[Baumgarten and Wieners, 2021] Baumgarten, N. and Wieners, C. (2021). The parallel finite element system M++ with integrated multilevel preconditioning and multilevel Monte Carlo methods. Computers \&5 Mathematics with Applications, 81:391-406.

[Bilgen and Weinberg, 2019] Bilgen, C. and Weinberg, K. (2019). On the crack-driving force of phase-field models in linearized and finite elasticity. Computer Methods in Applied Mechanics and Engineering (CMAME), 353:348-372.

[Bohlen et al., 2020] Bohlen, T., Fernandez, M. R., Ernesti, J., Rheinbay, C., Rieder, A., and Wieners, C. (2020). Visco-acoustic full waveform seismic inversion: from a DG forward solver to a Newton-CG inverse solver. CRC 1173 Preprint 2020/4, Karlsruhe Institute of Technology https://www.waves.kit.edu/downloads/CRC1173_Preprint_2020-4.pdf. To appear in Computational Methods in Applied Mathematics.

[Borden et al., 2012] Borden, M., Verhoosel, C., Scott, M., Hughes, T., and Landis, C. (2012). A phase-field description of dynamic brittle fracture. Comput. Meth. Appl. Mech. Enging., 217:77-95.

[Bourdin et al., 2008] Bourdin, B., Francfort, G., and Marigo, J.-J. (2008). The variational approach to fracture. Journal of elasticity, 91(1-3):5-148.

[Dumbser and Käser, 2006] Dumbser, M. and Käser, M. (2006). An arbitrary high-order discontinuous Galerkin method for elastic waves on unstructured meshes - II. The three-dimensional isotropic case. Geophysical Journal International, 167(1):319-336.

[Francfort and Marigo, 1998] Francfort, G. and Marigo, J.-J. (1998). Revisiting brittle fracture as an energy minimization problem. Journal of the Mechanics and Physics of Solids, 46:1319-1342.

[Freund, 1998] Freund, L. (1998). Dynamic fracture mechanics. Cambridge University Press.

[Griffith, 1921] Griffith, A. (1921). The phenomena of rupture and flow in solids. Philosophical Transactions of the Royal Society London, 221:163-198.

[Henry and Levine, 2004] Henry, H. and Levine, H. (2004). Dynamic instabilities of fracture under biaxial strain using a phase field model. Physics Review Letters, 93:105505.

[Hesch et al., 2017] Hesch, C., Gil, A., Ortigosa, R., Dittmann, M., Bilgen, C., Betsch, P., Franke, M., Janz, A., and Weinberg, K. (2017). A framework for polyconvex large strain phase-field methods to fracture. Computer Methods in Applied Mechanics and Engineering (CMAME), 317:649-683.

[Hesch and Weinberg, 2014] Hesch, C. and Weinberg, K. (2014). Thermodynamically consistent algorithms for a finite-deformation phase-field approach to fracture. International Journal for Numerical Methods in Engineering, 99(12):906-924.

[Hesthaven and Warburton, 2008] Hesthaven, J. S. and Warburton, T. (2008). Nodal Discontinuous Galerkin Methods. Springer.

[Hochbruck et al., 2015] Hochbruck, M., Pažur, T., Schulz, A., Thawinan, E., and Wieners, C. (2015). Efficient time integration for discontinuous Galerkin approximations of linear wave equations. ZAMM, 95(3):237-259. 
[Hughes, 1987] Hughes, T. (1987). The Finite Element Method, linear static and dynamic finite element analysis. Prentice-Hall, Englewood Cliffs, N.J.

[Irwin, 1958] Irwin, G. (1958). Elasticity and plasticity: fracture. In Függe, S., editor, Encyclopedia of Physics.

[Kuhn et al., 2015] Kuhn, C., Schlüter, A., and Müller, R. (2015). On degradation functions in phase field fracture models. Computational Materials Science, 108:374-384.

[Khosravani et al., 2019] Khosravani, M. R., Wagner, P., Fröhlich, D, and Weinberg, K. (2019). Dynamic fracture investigations of ultra-high performance concrete by spalling tests. Engineering Structures, 201:109844.

[Larsen et al., 2010] Larsen, C. J., Ortner, C., and Süli, E. (2010). Existence of solutions to a regularized model of dynamic fracture. Mathematical Models and Methods in Applied Sciences, 20(07):1021-1048.

[Mandal et al., 2020] Mandal, T. K., Nguyen, V. P., and Wu, J.-Y. (2020). Evaluation of variational phase-field models for dynamic brittle fracture. Engineering Fracture Mechanics, 235:107169.

[Miehe et al., 2010] Miehe, C., Hofacker, M., and Welschinger, F. (2010). A phase field model for rate-independent crack propagation: Robust algorithmic implementation based on operator splits. Comput. Methods Appl. Mech. Engrg., 199:2765-2778.

[Negri, 2016] Negri, M. (2016). Quasi-static evolutions in brittle fracture generated by gradient flows: sharp crack and phase-field approaches. Innovative Numerical Approaches for Multi-Field and Multi-Scale Problems, 81:197-216.

[Pandolfi et al., 2021] Pandolfi, A., Weinberg, K., and Ortiz, M. (2021). A comparative accuracy and convergence study of eigenerosion and phase-field models of fracture.

[Ren et al., 2019] Ren, H., Zhuang, X., Anitescu, C., and Rabczuk, T. (2019). An explicit phase field method for brittle dynamic fracture. Computers \& Structures, 217:45-56.

[Sargado et al., 2018] Sargado, J. M., Keilegavlen, E., Berre, I., and Nordbotten, J. M. (2018). High-accuracy phase-field models for brittle fracture based on a new family of degradation functions. Journal of the Mechanics and Physics of Solids, 111:458-489.

[Thomas et al., 2020] Thomas, M., Bilgen, C., and Weinberg, K. (2020). Analysis and simulations for a phase-field fracture model at finite strains based on modified invariants. Zeit. angew. Math. Mech. onlinelibrary.wiley.com/doi/pdfdirect/10.1002/zamm.201900288.

[Thomas and Tornquist, 2021] Thomas, M. and Tornquist, S. (2021). Discrete approximation of dynamic phase-field fracture in visco-elastic materials. Discrete \&S Continuous Dynamical Systems-S. https://www.aimsciences.org/article/doi/10.3934/ dcdss. 2021067.

[Weinberg et al., 2016] Weinberg, K., Dally, T., Schuß, S., Werner, M., and Bilgen, C. (2016). Modeling and numerical simulation of crack growth and damage with a phase field approach. GAMM-Mitteilungen, 39(1):55-77.

[Weinberg and Khosravani, 2018] Weinberg, K. and Khosravani, M. R (2018). On the tensile resistance of UHPC at impact. Eur. Phys. J. Special Topics, 227:167-177.

[Wieners, 2010] Wieners, C. (2010). A geometric data structure for parallel finite elements and the application to multigrid methods with block smoothing. Comput. Visual. Sci., 13:161-175. 